\title{
Dynamic Response of a Circular Tunnel in an Elastic Half Space
}

\author{
İran Coşkun and Demirhan Dolmaseven \\ Faculty of Civil Engineering, Yıldız Technical University, Davutpaşa Campus, Esenler, 34210 İstanbul, Turkey \\ Correspondence should be addressed to İrfan Coşkun; coskun@yildiz.edu.tr
}

Received 17 November 2016; Revised 1 March 2017; Accepted 9 March 2017; Published 26 April 2017

Academic Editor: Mohamed Ichchou

Copyright (C) 2017 Irfan Coşkun and Demirhan Dolmaseven. This is an open access article distributed under the Creative Commons Attribution License, which permits unrestricted use, distribution, and reproduction in any medium, provided the original work is properly cited.

\begin{abstract}
The vibration of a circular tunnel in an elastic half space subjected to uniformly distributed dynamic pressure at the inner boundary is studied in this paper. For comparison purposes, two different ground materials (soft and hard soil) are considered for the half space. Under the assumption of plane strain, the equations of motion for the tunnel and the surrounding medium are reduced to two wave equations in polar coordinates using Helmholtz potentials. The method of wave expansion is used to construct the displacement fields in terms of displacement potentials. The boundary conditions associated with the problem are satisfied exactly at the inner surface of the tunnel and at the interface between the tunnel and surrounding medium, and they are satisfied approximately at the free surface of the half space. A least-squares technique is used for satisfying the stress-free boundary conditions at the half space. It is shown by comparison that the stresses and displacements are significantly influenced by the properties of the surrounding soil, wave number (i.e., the frequency), depth of embedment, and thickness of the tunnel wall.
\end{abstract}

\section{Introduction}

The dynamic behavior of underground structures such as tunnels and pipelines is an important engineering problem in the field of dynamic soil-structure interactions. Compared to the large volume of literature on the dynamic response of structures in infinite media, the corresponding problem in a half space has not received much attention. Even so, this problem needs analysis, as half spaces are always present in metropolitan areas. It is assumed that this limitation is mainly due to the difficulties in satisfying boundary conditions at the free surface of the ground. Thiruvenkatachar and Viswanathan [1] investigated the dynamic response of an elastic half space with a cylindrical cavity at a finite depth subjected to time-dependent surface tractions on the boundary of the cavity using a series of wave functions and the method of successive approximations. El-Akily and Datta $[2,3]$ studied the response of a circular cylindrical shell to disturbances in an elastic half space using matched asymptotic expansions and a successive reflection technique. Datta et al. [4] studied the dynamic response of a cylindrical pipe with a circular cross-section lying in a concentric cylindrical region buried in an elastic half space. In this study, the fields within each of the regions were expanded in a series of wave functions, and the unknown coefficients appearing in the solution were obtained by considering a finite number of terms in the series. In another study, Wong et al. [5] considered the twodimensional response of a tunnel with a noncircular crosssection embedded in an elastic half space using a technique involving wave function expansion in the half space combined with a finite element representation of the tunnel and its vicinity. Balendra et al. [6] studied the in-plane vibrations of a tunnel-soil-building system in a viscoelastic half space. The displacement fields were formulated by the method of wave function expansion, and the boundary conditions were satisfied only at a finite number of points along the traction-free surface, tunnel-soil interface, and soil-foundation interface. Therefore, the unknown coefficients of the wave function were obtained using a least-squares approach. Lee and Karl [7] studied the scattering and diffraction of plane waves by underground, circular, and cylindrical cavities at various depths in a half space. In this study, the authors used the Fourier-Bessel series and approximated the half space as a large-diameter elastic cylinder. Luco and de Barros $[8,9]$ studied the seismic response of a cylindrical shell embedded in a layered viscoelastic half space using a technique that 
combined an indirect integral representation for the exterior domain with a simplified shell theory for tunnel representation. Guan and Moore [10] studied the dynamic response of multiple cavities deeply buried in a viscoelastic medium that were subjected to moving or seismic loading using the Fourier-Bessel series. Bayıroğlu [11] studied the dynamic response of an elastic half space with a circular cylindrical shell using the finite element method. Stamos and Beskos [12] studied the dynamic response of an infinitely long cylindrical tunnel buried in a half space that was subjected to plane harmonic waves using a special boundary element method in the frequency domain. Davis et al. [13] studied the transverse response of cylindrical cavities and pipes embedded in a half space that were subjected to incident plane SV waves. The solution was obtained using the Fourier-Bessel series and a convex approximation of the half-space free surface. Yang et al. [14] studied the ground vibrations caused by trains moving in tunnels embedded in half space using the finite element method. Liang et al. [15] investigated the diffraction of incident plane SV waves by a circular cavity in a saturated poroelastic half space using a wave function series and a downward concave approximation of the half-space free surface. Jiang et al. [16] studied the scattering of plane waves by a cylindrical cavity with lining in a poroelastic half plane using the complex variable function method. Zhou et al. [17] studied the scattering of the elastic waves by a circular pipeline in a poroelastic medium using the wave function expansion method. Gupta et al. [18] studied the generation and propagation of vibrations from underground railways by performing a parametric study on the soil and tunnel parameters. Lin et al. [19] studied the dynamic response of a circular underground tunnel in an elastic half space subjected to incident plane Pwaves using an analytical solution scheme. Coşkun et al. [20] studied the vibration of an elastic half space with a cylindrical cavity subjected to a uniform harmonic pressure at the inner surface using the method of wave function expansion. Liu and Wang [21] studied the dynamic response of twin circular tunnels in a full space subjected to plane harmonic excitation using the complex variable method. Liu et al. [22] studied the scattering of plane waves by a shallow lined circular tunnel in an elastic half space using the complex variable method and the image technique. Hamad et al. [23] studied the dynamic interaction of two parallel tunnels embedded in a half space using a fully coupled approach and a superposition approach. More recently, Huang et al. [24] studied the nonlinear dynamic responses of circular tunnels buried in normal fault ground using the finite element method.

In this study, the dynamic response of a circular cylindrical tunnel embedded in an elastic half space is analyzed. The tunnel lies parallel to the plane free surface of the medium at a finite depth and is subjected to a harmonic normal pressure at the inner surface. By introducing potentials, the governing equations for the tunnel and surrounding medium are decoupled and reduced to Helmholtz equations, satisfied by the potentials. The series solution for these equations is obtained via the wave function expansion method. The boundary conditions at the inner surface of the tunnel and at the tunnel-soil interface are satisfied exactly; they are satisfied only approximately along the traction-free surface of the half space using

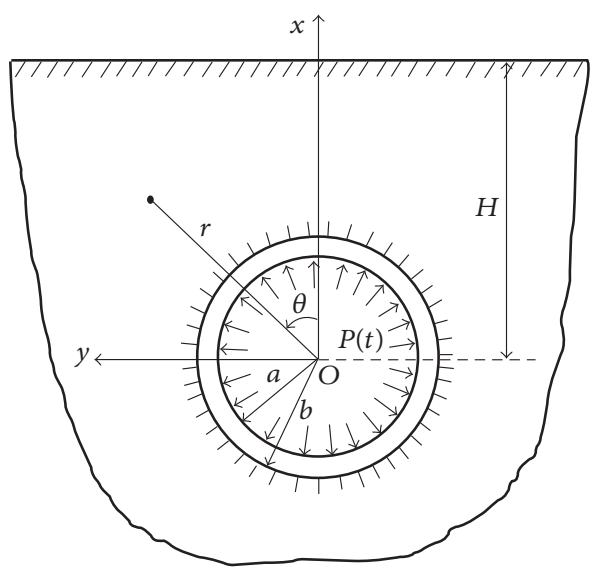

FIgure 1: Circular tunnel embedded in a half space.

the least-squares method. Once the unknown wave function coefficients are determined numerically, the displacements and stresses at any point in both the tunnel and surrounding medium can be calculated in a straightforward manner.

\section{Formulation of the Problem}

Consider an infinitely long circular tunnel with inner and outer radii $a$ and $b$, respectively, buried in an elastic half space at a depth $H$ below the free surface and subjected to harmonic pressure that is uniformly distributed as $P(t)=P_{0} e^{-i \omega t}$ at the inner surface (Figure 1). As the loading and geometry of the tunnel are assumed to be $z$-independent, the problem is twodimensional along the $x$ - and $y$-axis and corresponds to the plane strain case.

Therefore, using polar coordinates, the displacement vector $\mathbf{u}$ of a point will be a function of $r, \theta$, and $t$. The materials of the half space and the tunnel are assumed to be homogeneous, isotropic, and linearly elastic. The equation governing $\mathbf{u}$ is

$$
(\lambda+\mu)^{(\alpha)} \nabla \nabla \cdot \mathbf{u}^{(\alpha)}+\mu^{(\alpha)} \nabla^{2} \mathbf{u}^{(\alpha)}+\rho^{(\alpha)} \omega^{2} \mathbf{u}^{(\alpha)}=0,
$$

where $\lambda$ and $\mu$ are the Lamé constants, $\rho$ is the mass density, and $\omega$ is the circular frequency. The superscript $(\alpha)$ can be 1 or 2 depending on whether the point is in the tunnel or in the surrounding medium. It is assumed that the dependence on time is a simple harmonic of the form $e^{-i \omega t}$. For brevity, this term is henceforth suppressed from all expressions in the sequence. The displacement vector $\mathbf{u}$ can be written in terms of a dilatational scalar potential $\varphi(r, \theta)$ and an equivoluminal vector potential $\Psi(r, \theta)=\psi(r, \theta) \mathbf{e}_{z}$ with the form

$$
\mathbf{u}^{(\alpha)}=\nabla \varphi^{(\alpha)}+\nabla \times \Psi^{(\alpha)} ; \quad \nabla \cdot \Psi^{(\alpha)}=0,
$$

where $\mathbf{e}_{z}$ is the unit vector along the $z$-axis. Equation (2) is a solution for (1) if the potentials are solutions of the following reduced wave equations for steady-state, two-dimensional wave propagation:

$$
\begin{aligned}
& \nabla^{2} \varphi^{(\alpha)}+\left(k_{1}^{(\alpha)}\right)^{2} \varphi^{(\alpha)}=0 \\
& \nabla^{2} \psi^{(\alpha)}+\left(k_{2}^{(\alpha)}\right)^{2} \psi^{(\alpha)}=0
\end{aligned}
$$


where $k_{1}^{(\alpha)}$ and $k_{2}^{(\alpha)}$ are the longitudinal and shear wave numbers, respectively, and are given by

$$
\begin{aligned}
& k_{1}^{(\alpha)}=\frac{\omega}{\left\{\left(\lambda^{(\alpha)}+2 \mu^{(\alpha)}\right) / \rho^{(\alpha)}\right\}^{1 / 2}}, \\
& k_{2}^{(\alpha)}=\frac{\omega}{\left\{\mu^{(\alpha)} / \rho^{(\alpha)}\right\}^{1 / 2}} .
\end{aligned}
$$

The displacement vector can be written in terms of radial $u_{r}^{(\alpha)}$ and tangential $u_{\theta}^{(\alpha)}$ components as follows:

$$
\mathbf{u}^{(\alpha)}=u_{r}^{(\alpha)} \mathbf{e}_{r}+u_{\theta}^{(\alpha)} \mathbf{e}_{\theta},
$$

where $\mathbf{e}_{r}$ and $\mathbf{e}_{\theta}$ represent the unit vectors in the radial and tangential directions, respectively. Substitution of (5) into (2) yields

$$
\begin{aligned}
& u_{r}^{(\alpha)}=\frac{\partial \varphi^{(\alpha)}}{\partial r}+\frac{1}{r} \frac{\partial \psi^{(\alpha)}}{\partial \theta}, \\
& u_{\theta}^{(\alpha)}=\frac{1}{r} \frac{\partial \varphi^{(\alpha)}}{\partial \theta}-\frac{1}{r} \frac{\partial \psi^{(\alpha)}}{\partial r} .
\end{aligned}
$$

The corresponding stress components are related to the displacement via Hooke's law

$$
\begin{aligned}
& \sigma_{r r}^{(\alpha)}=\left(\lambda^{(\alpha)}+2 \mu^{(\alpha)}\right) \frac{\partial u_{r}^{(\alpha)}}{\partial r}+\frac{\lambda^{(\alpha)}}{r}\left(u_{r}^{(\alpha)}+\frac{\partial u_{\theta}^{(\alpha)}}{\partial \theta}\right) ; \\
& \sigma_{\theta \theta}^{(\alpha)}=\lambda^{(\alpha)} \frac{\partial u_{r}^{(\alpha)}}{\partial r}+\frac{1}{r}\left(\lambda^{(\alpha)}+2 \mu^{(\alpha)}\right)\left(u_{r}^{(\alpha)}+\frac{\partial u_{\theta}^{(\alpha)}}{\partial \theta}\right) ; \\
& \sigma_{r \theta}^{(\alpha)}=\frac{\mu^{(\alpha)}}{r}\left(r \frac{\partial u_{\theta}^{(\alpha)}}{\partial r}-u_{\theta}^{(\alpha)}+\frac{\partial u_{r}^{(\alpha)}}{\partial \theta}\right) .
\end{aligned}
$$

Using the method of separation and noting that the number $n$ must be an integer for a periodic solution, the solution of (3) can be written as [25]

$$
\begin{aligned}
& \varphi^{(\alpha)}=\sum_{n=-\infty}^{\infty}\left(\bar{A}_{n}^{(\alpha)} J_{n}\left(k_{1}^{(\alpha)} r\right)+\bar{B}_{n}^{(\alpha)} Y_{n}\left(k_{1}^{(\alpha)} r\right)\right) e^{i n \theta} \\
& \psi^{(\alpha)}=\sum_{n=-\infty}^{\infty}\left(\bar{C}_{n}^{(\alpha)} J_{n}\left(k_{2}^{(\alpha)} r\right)+\bar{D}_{n}^{(\alpha)} Y_{n}\left(k_{2}^{(\alpha)} r\right)\right) e^{i n \theta},
\end{aligned}
$$

where $J_{n}(\bullet)$ and $Y_{n}(\bullet)$ are Bessel functions of the first and second kind, order $n$, and $\bar{A}_{n}^{(\alpha)}, \bar{B}_{n}^{(\alpha)}, \bar{C}_{n}^{(\alpha)}$, and $\bar{D}_{n}^{(\alpha)}$ are constants. Using the relations $J_{-n}=(-1)^{n} J_{n}$ and $Y_{-n}=(-1)^{n} Y_{n}$ and considering the potentials $(\varphi)^{(\alpha)}$ and $(\psi)^{(\alpha)}$ to be symmetric and antisymmetric, respectively, (8) can be written in the form

$$
\begin{gathered}
\varphi^{(\alpha)}=\sum_{n=0}^{\infty}\left(A_{n}^{(\alpha)} J_{n}\left(k_{1}^{(\alpha)} r\right)+B_{n}^{(\alpha)} Y_{n}\left(k_{1}^{(\alpha)} r\right)\right) \cos n \theta ; \\
\psi^{(\alpha)}=\sum_{n=0}^{\infty}\left(C_{n}^{(\alpha)} J_{n}\left(k_{2}^{(\alpha)} r\right)+D_{n}^{(\alpha)} Y_{n}\left(k_{2}^{(\alpha)} r\right)\right) \sin n \theta,
\end{gathered}
$$

where $A_{n}^{(\alpha)}, B_{n}^{(\alpha)}, C_{n}^{(\alpha)}$, and $D_{n}^{(\alpha)}$ are constants to be determined from the boundary conditions. Substituting (9) into (6), the displacement fields are obtained as

$$
\begin{aligned}
u_{r}^{(\alpha)} & =\sum_{n=0}^{\infty}\left\{A_{n}^{(\alpha)}\left(\frac{n}{r} J_{n}\left(k_{1}^{(\alpha)} r\right)-k_{1}^{(\alpha)} J_{n+1}\left(k_{1}^{(\alpha)} r\right)\right)\right. \\
+ & B_{n}^{(\alpha)}\left(\frac{n}{r} Y_{n}\left(k_{1}^{(\alpha)} r\right)-k_{1}^{(\alpha)} Y_{n+1}\left(k_{1}^{(\alpha)} r\right)\right) \\
+ & \left.C_{n}^{(\alpha)}\left(\frac{n}{r} J_{n}\left(k_{2}^{(\alpha)} r\right)\right)+D_{n}^{(\alpha)}\left(\frac{n}{r} Y_{n}\left(k_{2}^{(\alpha)} r\right)\right)\right\} \\
& \cdot \cos n \theta, \\
u_{\theta}^{(\alpha)} & =\sum_{n=0}^{\infty}\left\{-A_{n}^{(\alpha)}\left(\frac{n}{r} J_{n}\left(k_{1}^{(\alpha)} r\right)\right)-B_{n}^{(\alpha)}\left(\frac{n}{r} Y_{n}\left(k_{1}^{(\alpha)} r\right)\right)\right. \\
& -C_{n}^{(\alpha)}\left(\frac{n}{r} J_{n}\left(k_{2}^{(\alpha)} r\right)-k_{2}^{(\alpha)} J_{n+1}\left(k_{2}^{(\alpha)} r\right)\right) \\
& \left.-D_{n}^{(\alpha)}\left(\frac{n}{r} Y_{n}\left(k_{2}^{(\alpha)} r\right)-k_{2}^{(\alpha)} Y_{n+1}\left(k_{2}^{(\alpha)} r\right)\right)\right\} \sin n \theta .
\end{aligned}
$$

Likewise, substituting (10) into (7), the associated stress fields can be written as

$$
\begin{aligned}
\sigma_{r r}^{(\alpha)} & =\sum_{n=0}^{\infty} 2 \mu^{(\alpha)}\left\{A _ { n } ^ { ( \alpha ) } \left(\beta_{1}^{(\alpha)} J_{n}\left(k_{1}^{(\alpha)} r\right)\right.\right. \\
& \left.+\frac{k_{1}^{(\alpha)}}{r} J_{n+1}\left(k_{1}^{(\alpha)} r\right)\right)+B_{n}^{(\alpha)}\left(\beta_{1}^{(\alpha)} Y_{n}\left(k_{1}^{(\alpha)} r\right)\right. \\
& \left.+\frac{k_{1}^{(\alpha)}}{r} Y_{n+1}\left(k_{1}^{(\alpha)} r\right)\right)+C_{n}^{(\alpha)}\left(\frac{n^{2}-n}{r^{2}} J_{n}\left(k_{2}^{(\alpha)} r\right)\right. \\
& \left.-\frac{k_{2}^{(\alpha)} n}{r} J_{n+1}\left(k_{2}^{(\alpha)} r\right)\right)+D_{n}^{(\alpha)}\left(\frac{n^{2}-n}{r^{2}} Y_{n}\left(k_{2}^{(\alpha)} r\right)\right. \\
& \left.\left.-\frac{k_{2}^{(\alpha)} n}{r} Y_{n+1}\left(k_{2}^{(\alpha)} r\right)\right)\right\} \cos n \theta, \\
\sigma_{\theta \theta}^{(\alpha)} & =\sum_{n=0}^{\infty} 2 \mu^{(\alpha)}\left\{A _ { n } ^ { ( \alpha ) } \left(\beta_{2}^{(\alpha)} J_{n}\left(k_{1}^{(\alpha)} r\right)\right.\right. \\
& \left.-\frac{k_{1}^{(\alpha)}}{r} J_{n+1}\left(k_{1}^{(\alpha)} r\right)\right)+B_{n}^{(\alpha)}\left(\beta_{2}^{(\alpha)} Y_{n}\left(k_{1}^{(\alpha)} r\right)\right. \\
& \left.-\frac{k_{1}^{(\alpha)}}{r} Y_{n+1}\left(k_{1}^{(\alpha)} r\right)\right)+C_{n}^{(\alpha)}\left(-\frac{n^{2}-n}{r^{2}} J_{n}\left(k_{2}^{(\alpha)} r\right)\right. \\
& \left.+\frac{k_{2}^{(\alpha)} n}{r} J_{n+1}\left(k_{2}^{(\alpha)} r\right)\right)+D_{n}^{(\alpha)}\left(-\frac{n^{2}-n}{r^{2}} Y_{n}\left(k_{2}^{(\alpha)} r\right)\right. \\
r & \left.\left.k_{n+1}^{(\alpha)}\left(k_{2}^{(\alpha)} r\right)\right)\right\} \cos n \theta \\
&
\end{aligned}
$$




$$
\begin{aligned}
\sigma_{r \theta}^{(\alpha)} & =\sum_{n=0}^{\infty} 2 \mu^{(\alpha)}\left\{A _ { n } ^ { ( \alpha ) } \left(-\frac{n^{2}-n}{r^{2}} J_{n}\left(k_{1}^{(\alpha)} r\right)\right.\right. \\
& \left.+\frac{k_{1}^{(\alpha)} n}{r} J_{n+1}\left(k_{1}^{(\alpha)} r\right)\right) \\
& +B_{n}^{(\alpha)}\left(-\frac{n^{2}-n}{r^{2}} Y_{n}\left(k_{1}^{(\alpha)} r\right)+\frac{k_{1}^{(\alpha)} n}{r} Y_{n+1}\left(k_{1}^{(\alpha)} r\right)\right) \\
& +C_{n}^{(\alpha)}\left(\beta_{3}^{(\alpha)} J_{n}\left(k_{2}^{(\alpha)} r\right)-\frac{k_{2}^{(\alpha)}}{r} J_{n+1}\left(k_{2}^{(\alpha)} r\right)\right) \\
+ & \left.D_{n}^{(\alpha)}\left(\beta_{3}^{(\alpha)} Y_{n}\left(k_{2}^{(\alpha)} r\right)-\frac{k_{2}^{(\alpha)}}{r} Y_{n+1}\left(k_{2}^{(\alpha)} r\right)\right)\right\}
\end{aligned}
$$

$\cdot \sin n \theta$,

where $\beta_{1}^{(\alpha)}=\left(n^{2}-n\right) / r^{2}-\left(\left(\lambda^{(\alpha)}+2 \mu^{(\alpha)}\right) / 2 \mu^{(\alpha)}\right)\left(k_{1}^{(\alpha)}\right)^{2}$, $\beta_{2}^{(\alpha)}=-\left(n^{2}-n\right) / r^{2}-\left(\lambda^{(\alpha)} / 2 \mu^{(\alpha)}\right)\left(k_{1}^{(\alpha)}\right)^{2}$, and $\beta_{3}^{(\alpha)}=-\left(n^{2}-n\right) /$ $r^{2}+(1 / 2)\left(k_{2}^{(\alpha)}\right)^{2}$.

The boundary conditions for the problem will be determined at the inner surface of the tunnel, at the interface between the tunnel and the surrounding medium, and at the free surface of the half space. It will be assumed that all contacts are perfect such that the displacements and the tractions are continuous across the interface between the tunnel and the surrounding medium. Given that the tunnel is subjected to an internal pressure $P_{0}$ in magnitude, the boundary conditions at $r=a$ are as follows:

$$
\begin{aligned}
& \sigma_{r r}^{(1)}=-P_{0}, \\
& \sigma_{r \theta}^{(1)}=0 .
\end{aligned}
$$

For the interface, at $r=b$,

$$
\begin{aligned}
& u_{r}^{(1)}=u_{r}^{(2)}, \\
& u_{\theta}^{(1)}=u_{\theta}^{(2)}, \\
& \sigma_{r r}^{(1)}=\sigma_{r r}^{(2)}, \\
& \sigma_{r \theta}^{(1)}=\sigma_{r \theta}^{(2)} .
\end{aligned}
$$

For the traction-free surface, at $x=H$,

$$
\begin{aligned}
& \sigma_{x x}^{(2)}=0, \\
& \sigma_{x y}^{(2)}=0 .
\end{aligned}
$$

To use the stress boundary conditions given in (16) and describe the displacement distribution on the free surface, the stress and displacement components are transformed into Cartesian coordinates via the relations

$$
\begin{aligned}
\sigma_{x x}^{(\alpha)}= & \sigma_{r r}^{(\alpha)} \cos ^{2} \theta+\sigma_{\theta \theta}^{(\alpha)} \sin ^{2} \theta+2 \sigma_{r \theta}^{(\alpha)} \sin \theta \cos \theta ; \\
\sigma_{x y}^{(\alpha)}= & -\left(\sigma_{r r}^{(\alpha)}-\sigma_{\theta \theta}^{(\alpha)}\right) \sin \theta \cos \theta \\
& +\sigma_{r \theta}^{(\alpha)}\left(\cos ^{2} \theta-\sin ^{2} \theta\right) ; \\
u_{x}^{(\alpha)}= & u_{r}^{(\alpha)} \cos \theta-u_{\theta}^{(\alpha)} \sin \theta ; \\
u_{y}^{(\alpha)}= & u_{r}^{(\alpha)} \sin \theta+u_{\theta}^{(\alpha)} \cos \theta .
\end{aligned}
$$

Substituting (11), (12), and (13) into (17a) and (17b) and (10) into (17c) and (17d), the transformed stress and displacement components can be obtained:

$$
\begin{aligned}
& \sigma_{x x}^{(\alpha)}=\sum_{n=0}^{\infty} 2 \mu^{(\alpha)}\left\langleA _ { n } ^ { ( \alpha ) } \left\{\left[-\frac{\lambda^{(\alpha)}+\mu^{(\alpha)}}{2 \mu^{(\alpha)}}\left(k_{1}^{(\alpha)}\right)^{2} J_{n}\left(k_{1 r}\right)+\left(\left(\frac{n^{2}-n}{r^{2}}-\frac{1}{2}\left(k_{1}^{(\alpha)}\right)^{2}\right) J_{n}\left(k_{1 r}\right)+\frac{k_{1}^{(\alpha)}}{r} J_{n+1}\left(k_{1 r}\right)\right) \cos 2 \theta\right]\right.\right. \\
& \left.\cdot \cos n \theta-\left[-\frac{n^{2}-n}{r^{2}} J_{n}\left(k_{1 r}\right)+\frac{k_{1}^{(\alpha)} n}{r} J_{n+1}\left(k_{1 r}\right)\right] \sin 2 \theta \sin n \theta\right\}+B_{n}^{(\alpha)}\{\bullet\}+C_{n}^{(\alpha)}\left\{\left[\frac{n^{2}-n}{r^{2}} J_{n}\left(k_{2 r}\right)-\frac{k_{2}^{(\alpha)} n}{r} J_{n+1}\left(k_{2 r}\right)\right]\right. \\
& \left.\left.\cdot \cos 2 \theta \cos n \theta-\left[\beta_{3}^{(\alpha)} J_{n}\left(k_{2 r}\right)-\frac{k_{2}^{(\alpha)}}{r} J_{n+1}\left(k_{2 r}\right)\right] \sin 2 \theta \sin n \theta\right\}+D_{n}^{(\alpha)}\{\bullet\}\right\rangle \\
& \sigma_{x y}^{(\alpha)}=\sum_{n=0}^{\infty} 2 \mu^{(\alpha)}\left\langleA _ { n } ^ { ( \alpha ) } \left\{\left[\left(\frac{n^{2}-n}{r^{2}}-\frac{1}{2}\left(k_{1}^{(\alpha)}\right)^{2}\right) J_{n}\left(k_{1 r}\right)+\frac{k_{1}^{(\alpha)}}{r} J_{n+1}\left(k_{1 r}\right)\right] \sin 2 \theta \cos n \theta\right.\right. \\
& \left.+\left[-\frac{n^{2}-n}{r^{2}} J_{n}\left(k_{1 r}\right)+\frac{k_{1}^{(\alpha)} n}{r} J_{n+1}\left(k_{1 r}\right)\right] \cos 2 \theta \sin n \theta\right\}+B_{n}^{(\alpha)}\{\bullet\} \\
& +C_{n}^{(\alpha)}\left\{\left[\frac{n^{2}-n}{r^{2}} J_{n}\left(k_{2 r}\right)-\frac{k_{2}^{(\alpha)} n}{r} J_{n+1}\left(k_{2 r}\right)\right] \sin 2 \theta \cos n \theta+\left[\beta_{3}^{(\alpha)} J_{n}\left(k_{2 r}\right)-\frac{k_{2}^{(\alpha)}}{r} J_{n+1}\left(k_{2 r}\right)\right] \cos 2 \theta \sin n \theta\right\} \\
& \left.+D_{n}^{(\alpha)}\{\bullet\}\right\rangle
\end{aligned}
$$




$$
\begin{aligned}
u_{x}^{(\alpha)} & =\sum_{n=0}^{\infty}\left\langle A_{n}^{(\alpha)}\left\{\left[\frac{n}{r} J_{n}\left(k_{1 r}\right)-k_{1}^{(\alpha)} J_{n+1}\left(k_{1 r}\right)\right] \cos \theta \cos n \theta+\frac{n}{r} J_{n}\left(k_{1 r}\right) \sin \theta \sin n \theta\right\}+B_{n}^{(\alpha)}\{\bullet\}\right. \\
& \left.+C_{n}^{(\alpha)}\left\{\left[\frac{n}{r} J_{n}\left(k_{2 r}\right)-k_{2}^{(\alpha)} J_{n+1}\left(k_{2 r}\right)\right] \sin \theta \sin n \theta+\frac{n}{r} J_{n}\left(k_{2 r}\right) \cos \theta \cos n \theta\right\}+D_{n}^{(\alpha)}\{\bullet\}\right\rangle, \\
u_{y}^{(\alpha)} & =\sum_{n=0}^{\infty}\left\langle A_{n}^{(\alpha)}\left\{\left[\frac{n}{r} J_{n}\left(k_{1 r}\right)-k_{1}^{(\alpha)} J_{n+1}\left(k_{1 r}\right)\right] \sin \theta \cos n \theta-\frac{n}{r} J_{n}\left(k_{1 r}\right) \cos \theta \sin n \theta\right\}+B_{n}^{(\alpha)}\{\bullet\}\right. \\
& \left.+C_{n}^{(\alpha)}\left\{-\left[\frac{n}{r} J_{n}\left(k_{2 r}\right)-k_{2}^{(\alpha)} J_{n+1}\left(k_{2 r}\right)\right] \cos \theta \sin n \theta+\frac{n}{r} J_{n}\left(k_{2 r}\right) \sin \theta \cos n \theta\right\}+D_{n}^{(\alpha)}\{\bullet\}\right\rangle,
\end{aligned}
$$

where $k_{1 r}=k_{1}^{(\alpha)} r, k_{2 r}=k_{2}^{(\alpha)} r$, and $\{\bullet\}$ represents the coefficients of $B_{n}^{(\alpha)}$ and $D_{n}^{(\alpha)}$ having the same form as the coefficients of $A_{n}^{(\alpha)}$ and $C_{n}^{(\alpha)}$, respectively, and are obtained by replacing $J_{n}(\bullet)$ and $J_{n+1}(\bullet)$ with $Y_{n}(\bullet)$ and $Y_{n+1}(\bullet)$.

\section{Solution of the Problem}

Equations (10)-(13) constitute a system containing an infinite number of equations with the unknown constants $A_{n}^{(\alpha)}, B_{n}^{(\alpha)}$, $C_{n}^{(\alpha)}$, and $D_{n}^{(\alpha)}(n=0,1,2, \ldots)$. Because of the difficulty of taking into account an infinite number of terms in the series given above, particularly for the functions $Y_{n}$ for a small argument with a large index, the series are truncated at a finite number $N$. Furthermore, as the coefficients of $C_{n}^{(\alpha)}$ and $D_{n}^{(\alpha)}$ become zero for $n=0$ in (10)-(13), the constants $C_{0}^{(\alpha)}$ and $D_{0}^{(\alpha)}$ can be likewise assumed to be zero. In this case, the number of constants $\left(A_{0}^{(\alpha)}, B_{0}^{(\alpha)}, A_{n}^{(\alpha)}, B_{n}^{(\alpha)}, C_{n}^{(\alpha)}\right.$, and $\left.D_{n}^{(\alpha)} ; \alpha=1,2 ; n=1,2, \ldots, N\right)$ to be determined in the equations becomes $8 \times N+4$. To obtain these constants, one can use the boundary conditions given by (14) and (15). Substituting (11) into (14) and considering that the solution must be valid for all values of $\theta, N+1$ equations are obtained. This solution is obtained by equating the coefficient of $\cos n \theta$ to $P_{0}$ for $n=0$ and zero for $n>0$, respectively. Similarly, substituting (13) into (14) and equating the coefficient of $\sin n \theta$ to zero for $n>0, N$ equations are obtained. Therefore, using the boundary conditions at the inner surface of the tunnel $(r=a), 2 \times N+1$ equations are obtained. Following the same solution procedure, $4 \times N+2$ equations are obtained from the interface boundary conditions (i.e., (15)). Therefore, the total number of equations becomes $6 \times N+3$. The necessary $2 \times N+1$ equations will be obtained from the boundary conditions given by (16), which require the stresses at each point of the free surface to be equal to zero. As there are an infinite number of points along the surface, the use of these conditions leads to an infinite set of algebraic equations. Furthermore, these boundary conditions cannot be satisfied exactly at each point as the series are truncated by keeping only a finite number of terms in the summations. Therefore, the boundary conditions (i.e., (16)) are satisfied approximately only at a finite number of points along the free surface by applying the least-squares technique. For this purpose, using $4 \times N+2$ equations obtained for the interface, the constants of region $2(\alpha=2)$ are solved in terms of the $2 \times N+1$ constants of region $1(\alpha=1)$ as

$$
\begin{aligned}
& A_{0}^{(2)}=f\left(B_{0}^{(1)}\right), \\
& B_{0}^{(2)}=f\left(B_{0}^{(1)}\right), \\
& A_{n}^{(2)}=f\left(C_{n}^{(1)}, D_{n}^{(1)}\right), \\
& B_{n}^{(2)}=f\left(C_{n}^{(1)}, D_{n}^{(1)}\right), \\
& C_{n}^{(2)}=f\left(C_{n}^{(1)}, D_{n}^{(1)}\right) \\
& D_{n}^{(2)}=f\left(C_{n}^{(1)}, D_{n}^{(1)}\right),
\end{aligned}
$$

and then substituted into (18) and (19). This procedure reduces the number of constants to $2 \times N+1$ in these equations. Subsequently, these $2 \times N+1$ constants can be obtained from a set of algebraic equations that will be established by using $r_{k}=H / \cos \theta_{k}$ in (18) and (19) and applying the least-squares technique given by

$$
\Pi=\sum_{k=1}^{M}\left(\left(\sigma_{x x}^{(k)}-\bar{\sigma}_{x x}^{(k)}\right)^{2}+\left(\sigma_{x y}^{(k)}-\bar{\sigma}_{x y}^{(k)}\right)^{2}\right)=\min .
$$

Here, $M$ is the number of points on the free surface, $\sigma_{x x}^{(k)}$ and $\sigma_{x y}^{(k)}$ are the stress components to be computed approximately at any point $k$, and $\bar{\sigma}_{x x}^{(k)}$ and $\bar{\sigma}_{x y}^{(k)}$ are the external stress components at these points. Using (23), a set of algebraic equations for the unknowns $B_{0}^{(1)}, C_{n}^{(1)}$, and $D_{n}^{(1)}$ can be found from

$$
\begin{aligned}
\frac{\partial \Pi}{\partial B_{0}^{(1)}}=\sum_{k=1}^{M}\left(\sigma_{x x}^{(k)} \frac{\partial \sigma_{x x}^{(k)}}{\partial B_{0}^{(1)}}+\sigma_{x y}^{(k)} \frac{\partial \sigma_{x y}^{(k)}}{\partial B_{0}^{(1)}}\right) & =0, \\
\frac{\partial \Pi}{\partial C_{n}^{(1)}}=\sum_{k=1}^{M}\left(\sigma_{x x}^{(k)} \frac{\partial \sigma_{x x}^{(k)}}{\partial C_{n}^{(1)}}+\sigma_{x y}^{(k)} \frac{\partial \sigma_{x y}^{(k)}}{\partial C_{n}^{(1)}}\right) & =0, \\
n & =1,2, \ldots, N, \\
\frac{\partial \Pi}{\partial D_{n}^{(1)}}=\sum_{k=1}^{M}\left(\sigma_{x x}^{(k)} \frac{\partial \sigma_{x x}^{(k)}}{\partial D_{n}^{(1)}}+\sigma_{x y}^{(k)} \frac{\partial \sigma_{x y}^{(k)}}{\partial D_{n}^{(1)}}\right) & =0, \\
n & =1,2, \ldots, N .
\end{aligned}
$$


Here, it is assumed that $\bar{\sigma}_{x x}^{(k)}=\bar{\sigma}_{x y}^{(k)}=0$ at $r_{k}=H / \cos \theta_{k}$ as the surface is traction-free. After determining $2 \times N+1$ constants from (24), the constants $A_{0}^{(2)}, B_{0}^{(2)}, A_{n}^{(2)}, B_{n}^{(2)}, C_{n}^{(2)}$, and $D_{n}^{(2)}$ can be obtained from (22). Finally, the remaining constants $\left(A_{0}^{(1)}, A_{n}^{(1)}\right.$, and $\left.B_{n}^{(1)}\right)$ can be determined through (14) as they are dependent on the other constants of region 1. Once the constants are obtained, the displacement and stress components at any point can be calculated.

\section{Numerical Results and Discussion}

Numerical computations are presented for a concrete circular tunnel of outer radius $b=6 \mathrm{~m}$ buried in the elastic half space shown in Figure 1. The wall thickness of the tunnel and the inner radius are taken to be $t=t^{*} b$ and $a=(1-$ $\left.t^{*}\right) b$, respectively, where $t^{*}\left(0<t^{*}<1\right)$ represents the thickness parameter. The material properties for the tunnel are $\rho=2410 \mathrm{~kg} / \mathrm{m}^{3}, E=2.01 \times 10^{10} \mathrm{~N} / \mathrm{m}^{2}$, and $\nu=$ 0.2 . Two types of outside ground material are considered for comparison purposes. The first ground material is hard soil with properties $\rho^{(1)}=2664 \mathrm{~kg} / \mathrm{m}^{3}, E^{(1)}=7.569 \times 10^{9} \mathrm{~N} / \mathrm{m}^{2}$, and $v^{(1)}=0.333$. The second ground material is soft soil with properties $\rho^{(2)}=2665 \mathrm{~kg} / \mathrm{m}^{3}, E^{(2)}=6.9 \times 10^{8} \mathrm{~N} / \mathrm{m}^{2}$, and $v^{(2)}=0.45$. The internal pressure $P_{0}$ on the inner surface of the tunnel, the truncation constant $N$, and the region at the free surface are taken to be $P_{0}=10^{5} \mathrm{~N} / \mathrm{m}^{2}, N=8$, and $-30 \times$ $b \leq y \leq 30 \times b$, respectively. The dimensionless wave number $\left(k_{2}^{(2)} b\right)$, the dimensionless depth ratio $(H / b)$, and the thickness parameter $\left(t^{*}\right)$ are used to represent the behavior. Furthermore, the following dimensionless parameters are used to show numerical results: radial and tangential displacement amplitudes $u_{r}^{*}=\left|u_{r}\right| \lambda^{(1)} / b P_{0}$ and $u_{\theta}^{*}=\left|u_{\theta}\right| \lambda^{(1)} / b P_{0}$, surface displacement amplitudes $u_{x}^{*}=\left|u_{x}\right| \lambda^{(1)} / b P_{0}$, and radial and tangential stress amplitudes $\sigma_{r r}^{*}=\left|\sigma_{r r}\right| / P_{0}$ and $\sigma_{\theta \theta}^{*}=\left|\sigma_{\theta \theta}\right| / P_{0}$. Owing to the symmetry of the system with respect to the $x$-axis, the displacement and stress distributions become symmetrical about this axis in all figures.

Figures 2-5 show the effect of varying the wave number (i.e., the frequency) on the displacements and stresses at the outer surface of the tunnel wall when the surrounding material is hard soil. Note that $k_{2}^{(2)}=\omega / \beta_{2}^{(2)}$, in which $\beta_{2}^{(2)}$ is equal to the shear wave speed in the ground.

One can see from Figure 2 that radial displacements increase with frequency first and then decrease. Depending on the frequency, maximum radial displacements occur at $\theta=0^{\circ}$ and $\theta=180^{\circ}$, that is, at the points nearest and farthest from the free surface. As in the case of the radial displacements, tangential displacements (Figure 3) are found to increase first with frequency and then decrease. Their maximum values (except at low frequencies) occur at approximately $\theta= \pm 45^{\circ}$ and $\theta= \pm 120^{\circ}$. Figures 4 and 5 show the results for radial and tangential stresses, respectively. One can see from Figure 4 that the radial stress distribution is nearly symmetric about the center and that the stresses first decrease and then increase with increasing frequency. As in the case of the radial displacements, the maximum radial stresses occur at the points nearest and farthest from the free

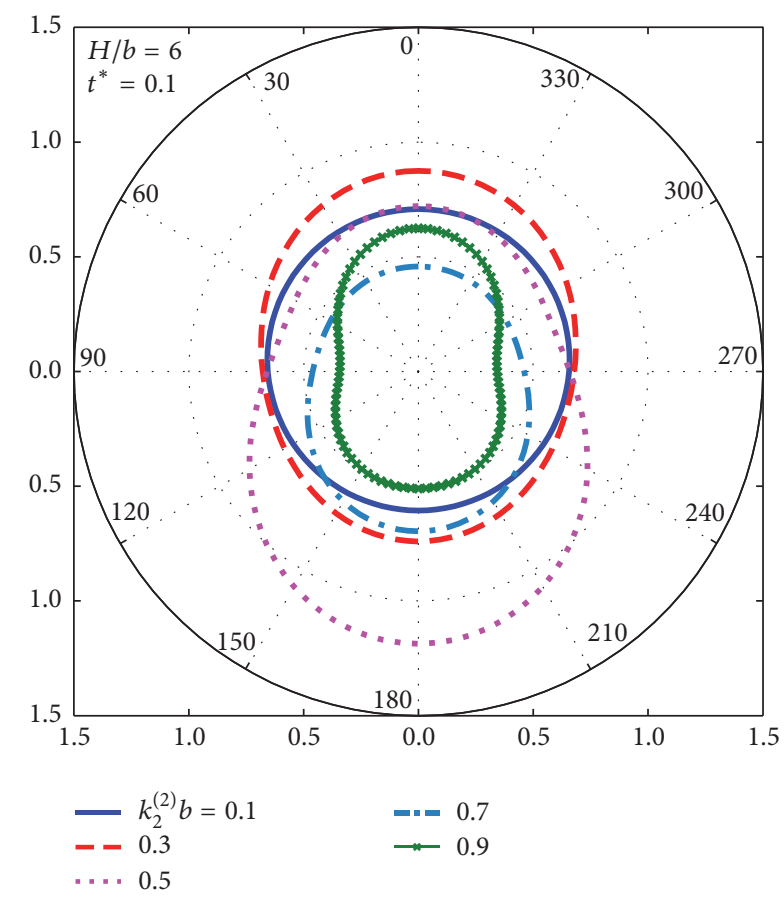

FIGURE 2: Radial displacement $\left(u_{r}^{*}\right)$ versus polar angle at $r=b$ for different wave numbers $\left(k_{2}^{(2)} b\right)$ for a tunnel in hard soil.

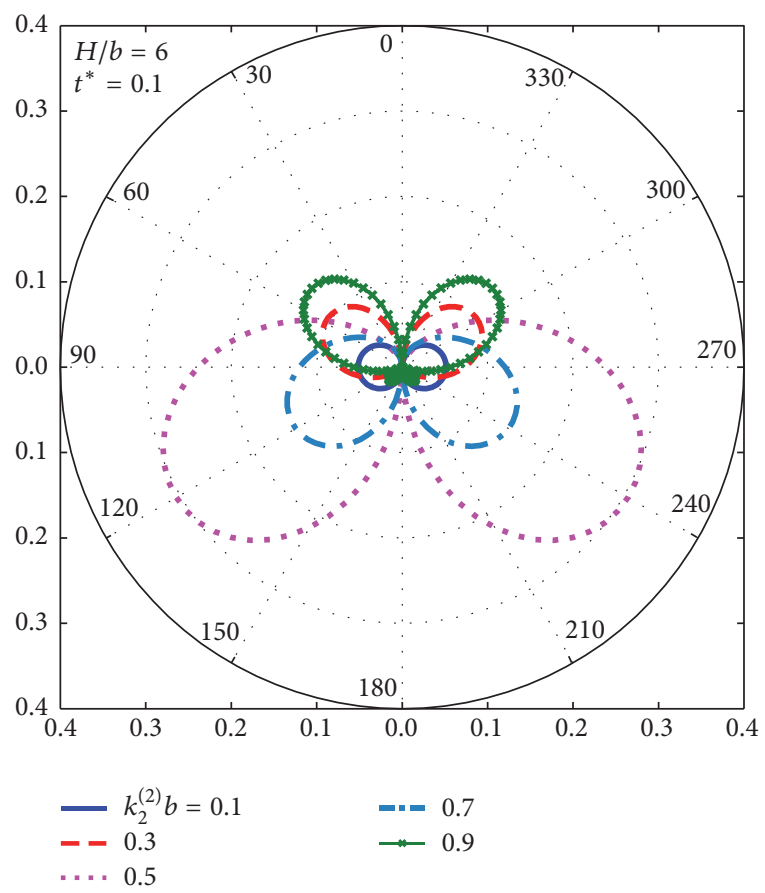

Figure 3: Tangential displacement $\left(u_{\theta}^{*}\right)$ versus polar angle at $r=b$ for different wave numbers $\left(k_{2}^{(2)} b\right)$ for a tunnel in hard soil.

surface. In contrast to the radial stresses, tangential stresses (Figure 5) first increase with frequency and then decrease; the maximum values occur at nearly $\theta= \pm 90^{\circ}$. Furthermore, at low frequencies the distribution is nearly symmetrical about the $y$-axis. However, as the frequency increased, the distribution became more asymmetric. From Figures 2-5, one can 


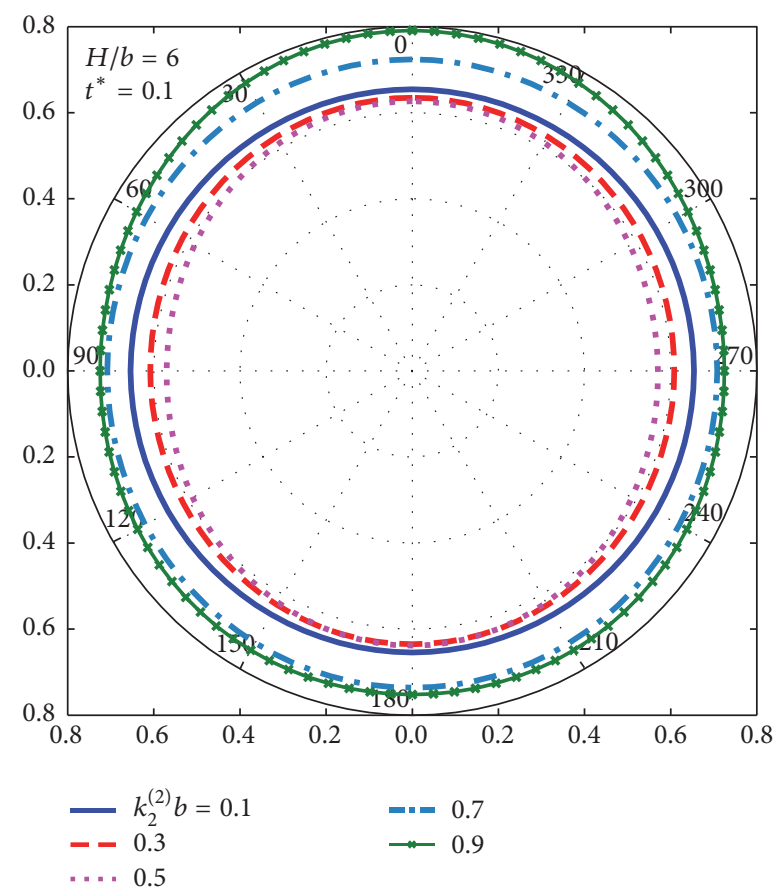

Figure 4: Radial stress $\left(\sigma_{r r}^{*}\right)$ versus polar angle at $r=b$ for different wave numbers $\left(k_{2}^{(2)} b\right)$ for a tunnel in hard soil.

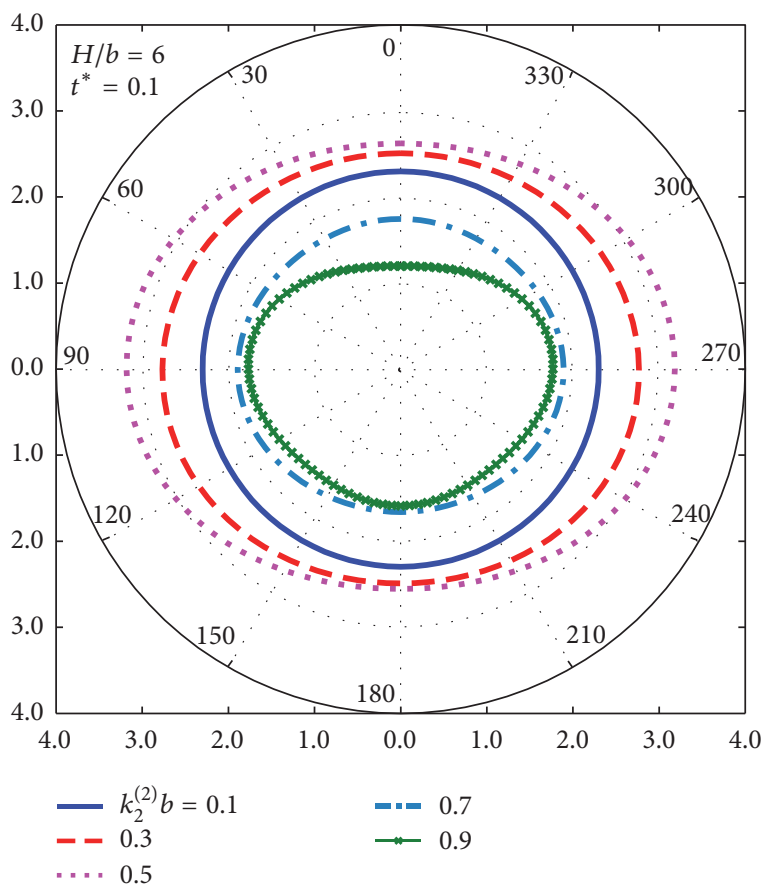

Figure 5: Tangential stress $\left(\sigma_{\theta \theta}^{*}\right)$ versus polar angle at $r=b$ for different wave numbers $\left(k_{2}^{(2)} b\right)$ for a tunnel in hard soil.

observe that the radial displacements and tangential stresses are considerably larger than the corresponding tangential displacements and radial stresses at the same frequency.

Figure 6 shows the variations of the radial displacement with respect to the frequency at $r=b$ for the top $\left(\theta=0^{\circ}\right)$ and bottom $\left(\theta=180^{\circ}\right)$ points of the tunnel. As in the spatial

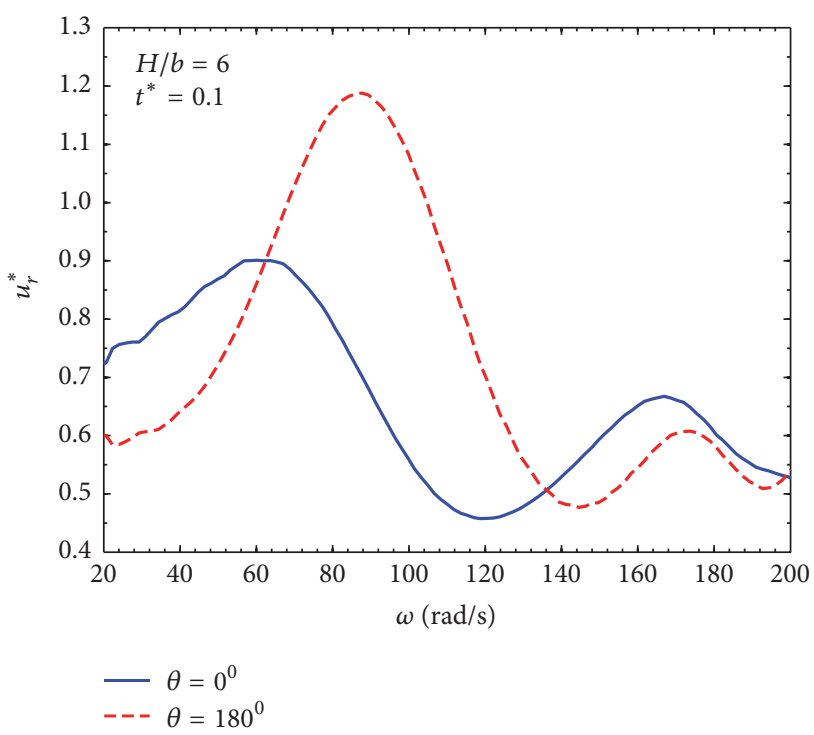

FIGURE 6: Radial displacement $\left(u_{r}^{*}\right)$ versus frequency at $r=b$ for $\theta=0^{\circ}$ and $\theta=180^{\circ}$ for a tunnel in hard soil.

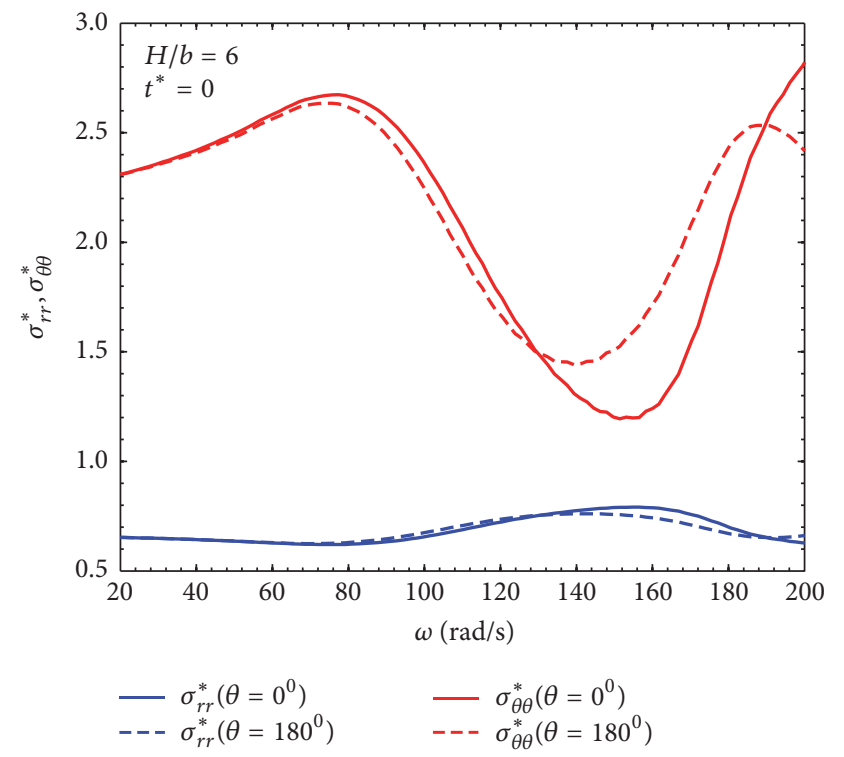

FIGURE 7: Radial $\left(\sigma_{r r}^{*}\right)$ and tangential $\left(\sigma_{\theta \theta}^{*}\right)$ stresses versus frequency at $r=b$ for $\theta=0^{\circ}$ and $\theta=180^{\circ}$ for a tunnel in hard soil.

distribution shown in Figure 2, radial displacement increases initially as the frequency increases and then decreases and increases again as the frequency increases further. The maximum value occurs at $\theta=180^{\circ}$, that is, at the point farthest from the free surface. Figure 7 shows the variations of the radial and tangential stresses with respect to the frequency at $r=b$ for the top $\left(\theta=0^{\circ}\right)$ and bottom $\left(\theta=180^{\circ}\right)$ points of the tunnel. One can see that the tangential stresses, as expected, are significantly higher than the radial stresses for all frequency values. In addition, the maximum values occur at the top point of the tunnel for both the radial and tangential stresses at different frequency values. 


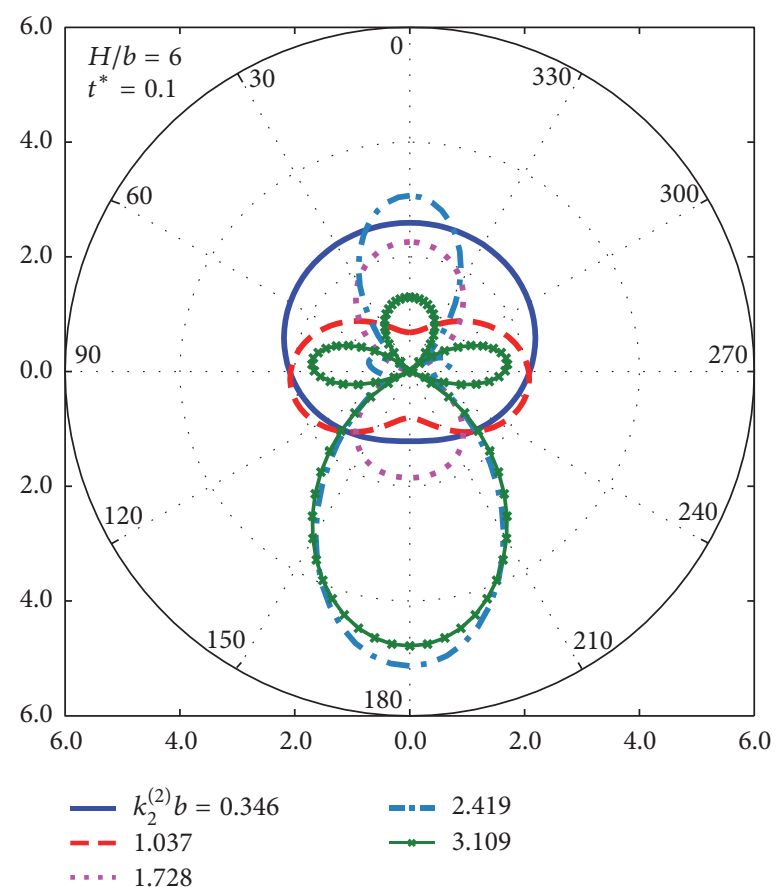

FIgURE 8: Radial displacement $\left(u_{r}^{*}\right)$ versus polar angle at $r=b$ for different wave numbers $\left(k_{2}^{(2)} b\right)$ for a tunnel in soft soil.

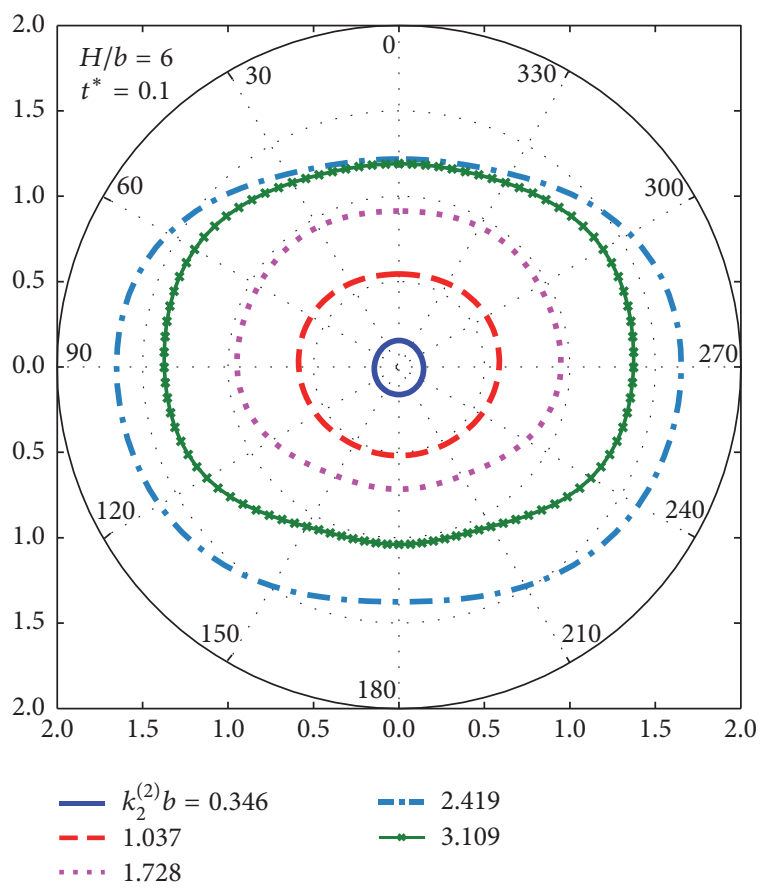

FIGURE 9: Radial stress $\left(\sigma_{r r}^{*}\right)$ versus polar angle at $r=b$ for different wave numbers $\left(k_{2}^{(2)} b\right)$ for a tunnel in soft soil.

Figures 8-10 show the results for a tunnel embedded in soft soil. Note that the ratio of the shear wave speeds in the hard and soft soils is 3.455. Because of this fact, the value of $k_{2}^{(2)} b$ in soft soil is larger by a factor of 3.455 compared with that for hard soil for the same frequency. Therefore, for

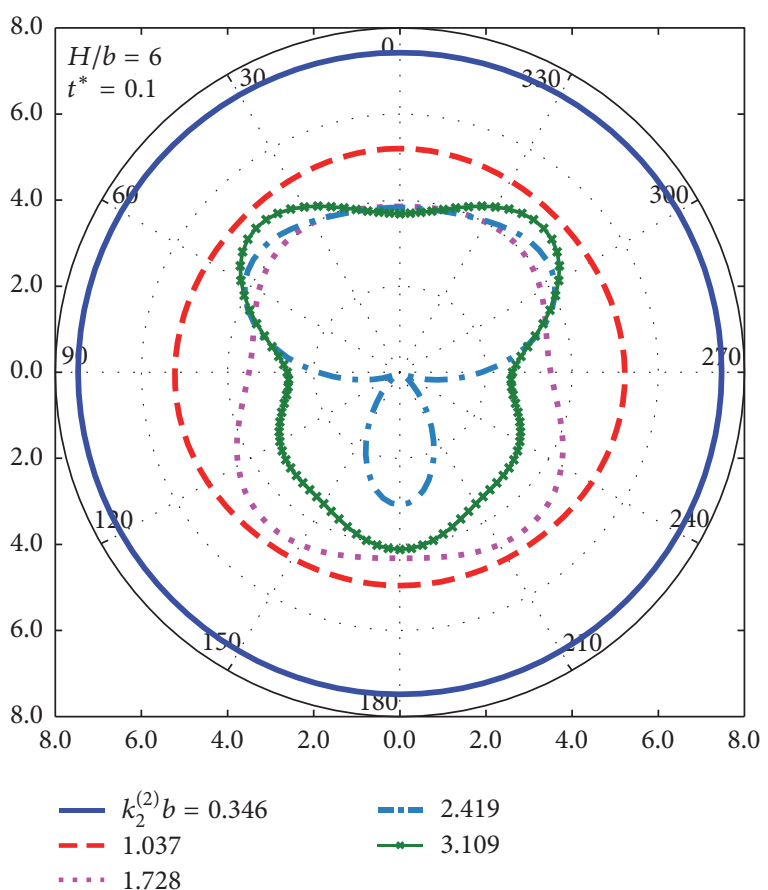

FIgURE 10: Tangential stress $\left(\sigma_{\theta \theta}^{*}\right)$ versus polar angle at $r=b$ for different wave numbers $\left(k_{2}^{(2)} b\right)$ for a tunnel in soft soil.

example, $k_{2}^{(2)} b=0.5$ in hard soil corresponds to $k_{2}^{(2)} b=1.728$ in soft soil. Radial displacements in the tunnel are shown in Figure 8. One can see that the displacement configuration has a complicated structure with local maxima and minima. However, as in the case of the hard soil, the maximum radial displacements occur at $\theta=0^{\circ}$ and $\theta=180^{\circ}$ except for $k_{2}^{(2)} b=$ 1.037 , for which the maximum occurs at nearly $\theta= \pm 90^{\circ}$. The maximum value decreases initially as the frequency increases and then increases and decreases again as the frequency increases further. Tangential displacements were also considered, but the results are not shown here. It was found that the maximum tangential displacement in the tunnel decreased first with frequency, then increased, and then decreased again, similar to the radial displacements. Radial stresses in the tunnel are shown in Figure 9. One can see that radial stresses increase with frequency first and then decrease. Maximum values occur in the upper half of the tunnel but close to $\pm 90^{\circ}$ at high frequencies. Note that the variations shown in Figure 9 are quite different from those shown in Figure 4. Tangential stresses in the tunnel are shown in Figure 10. One can see that the stress distribution is nearly symmetrical about the center at low frequencies. Stresses decrease initially as the frequency increases and then increase as the frequency increases further. As in the case of the hard soil, tangential stresses in soft soil are found to be considerably larger than radial stresses.

Figures 11 and 12 show the effect of varying the depth of the tunnel on the radial displacements at the tunnel wall for $k_{2}^{(2)} b=0.9$ and 3.109 in hard and soft soil, respectively. The corresponding frequency for these dimensionless wave numbers is $\omega=154.83 \mathrm{rad} / \mathrm{s}$. In hard soil (Figure 11), 


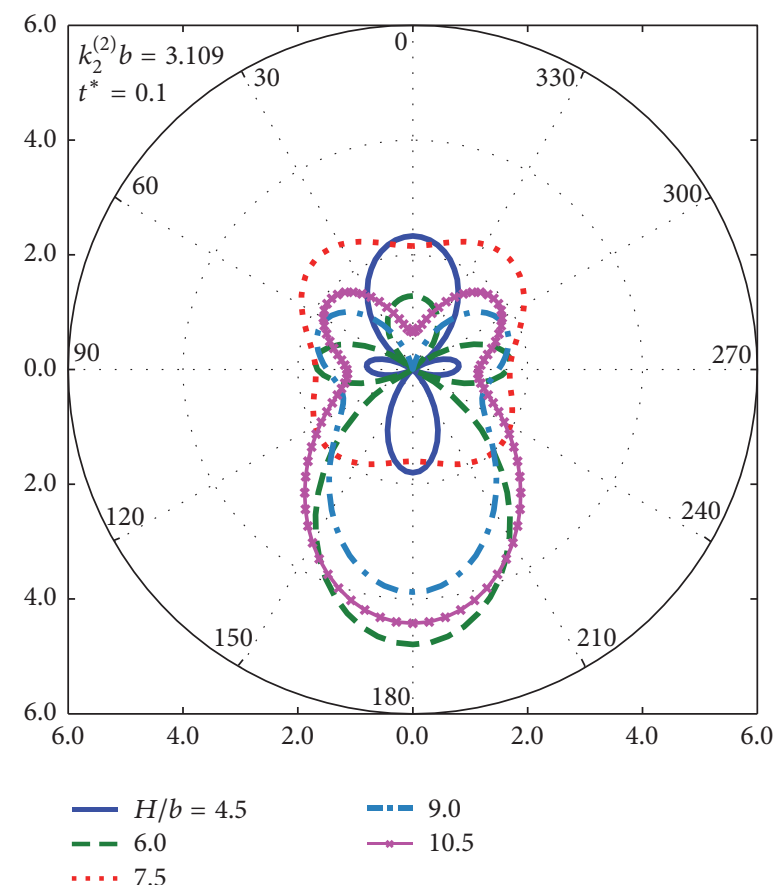

FIGURE 11: Radial displacement $\left(u_{r}^{*}\right)$ versus polar angle at $r=b$ for different depths $(H / b)$ for a tunnel in hard soil.

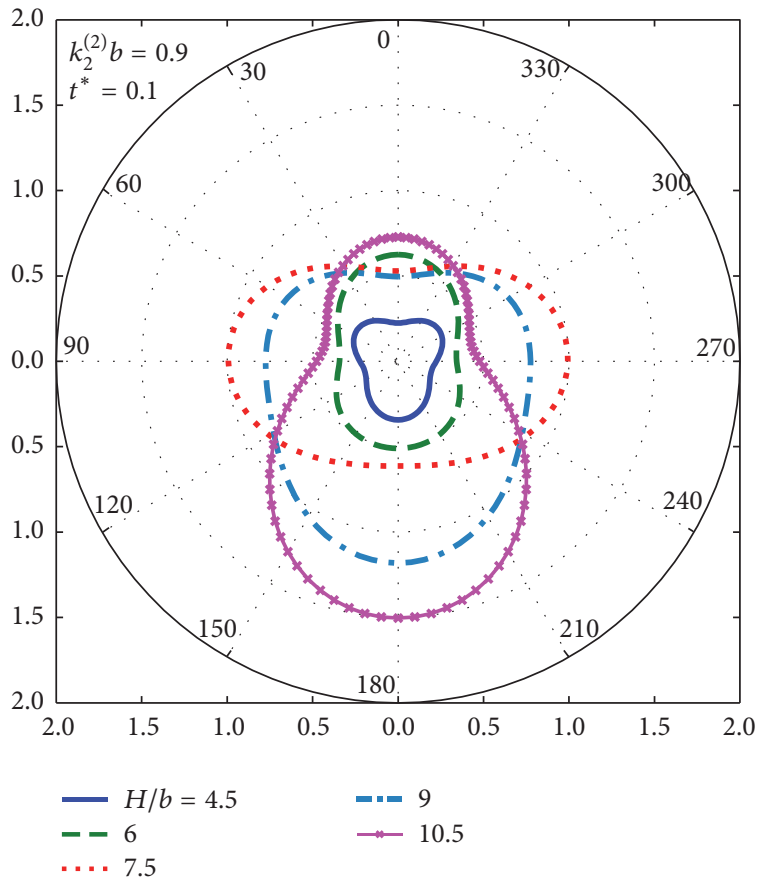

FIGURE 12: Radial displacement $\left(u_{r}^{*}\right)$ versus polar angle at $r=b$ for different depths $(H / b)$ for a tunnel in soft soil.

the maximum radial displacement, which increases in magnitude with depth continuously, occurs at the points nearest and farthest from the free surface (i.e., $\theta=0^{\circ}$ and $\theta=$ $180^{\circ}$ ). The exception is $H / b=7.5$, for which the maximum occurs at $\theta= \pm 90^{\circ}$. However, in soft soil (Figure 12) the maximum radial displacement increases initially as depth

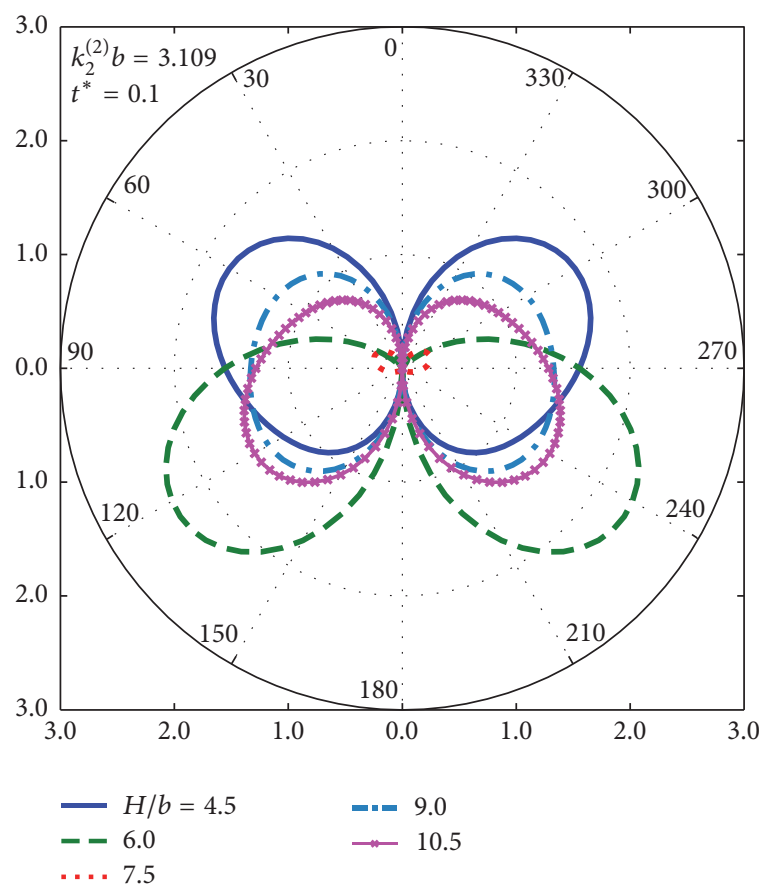

Figure 13: Tangential displacement $\left(u_{\theta}^{*}\right)$ versus polar angle at $r=b$ for different depths $(H / b)$ for a tunnel in soft soil.

increases and then decreases and increases again as the depth increases further. The same behavior is observed for the maximum tangential displacement (Figure 13). As can be seen in Figure 12, maximum radial displacements occur at $\theta=0^{\circ}$ and $\theta=180^{\circ}$. The exception is $H / b=7.5$ for which the maximum occurs at around $\theta= \pm 45^{\circ}$. On the other hand, the maximum tangential displacement in hard soil (not shown here) was found to increase with depth.

Figures 14 and 15 show the effect of varying the depth of the tunnel on the tangential stresses at the tunnel wall in hard and soft soil, respectively. The wave numbers are the same as noted previously. In hard soil (Figure 14), the maximum tangential stress, which increases in magnitude with depth, is found to occur at $\theta=0^{\circ}$ and $\theta= \pm 90^{\circ}$. However, the maximum value in soft soil (Figure 15) increases first with depth, then decreases, and then increases again, like in the radial displacements. Note that the maximum tangential stresses occur at $\theta= \pm 45^{\circ}$, except for $H / b=4.5$. In contrast to the maximum tangential stresses, maximum radial stresses (not shown here) decreased in magnitude continuously with increasing depth for both of soils. This decrease, however, occurred slowly in the hard soil but sharply in the soft soil. Furthermore, numerical results revealed that the tangential stresses and their maximum values increased slightly for both soils if the depth was increased further beyond the values that we examined. Based on the results in Figures 2-15 for two different ground materials, one can observe that larger stresses and displacements occur in tunnels embedded in soft soil.

Figure 16 shows the effect of varying the wall thickness of the tunnel on the tangential stresses for the hard-soil case. One can see that the maximum stress, which occurs at nearly $\theta= \pm 90^{\circ}$, decreases as the thickness of the tunnel increases. 


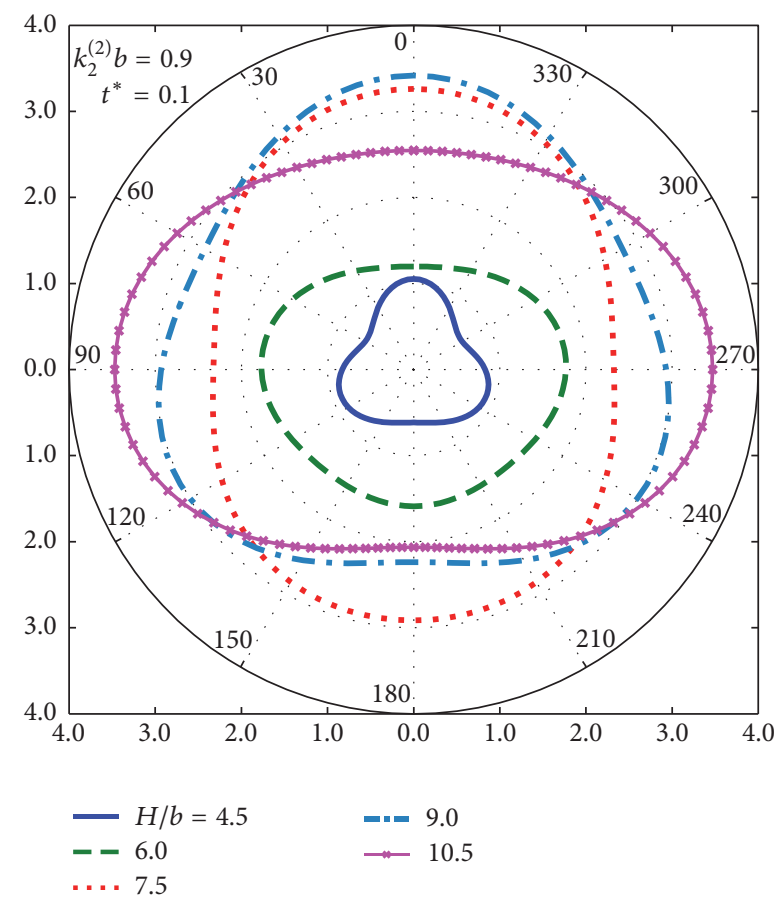

FIgURE 14: Tangential stress $\left(\sigma_{\theta \theta}^{*}\right)$ versus polar angle at $r=b$ for different depths $(H / b)$ for a tunnel in hard soil.

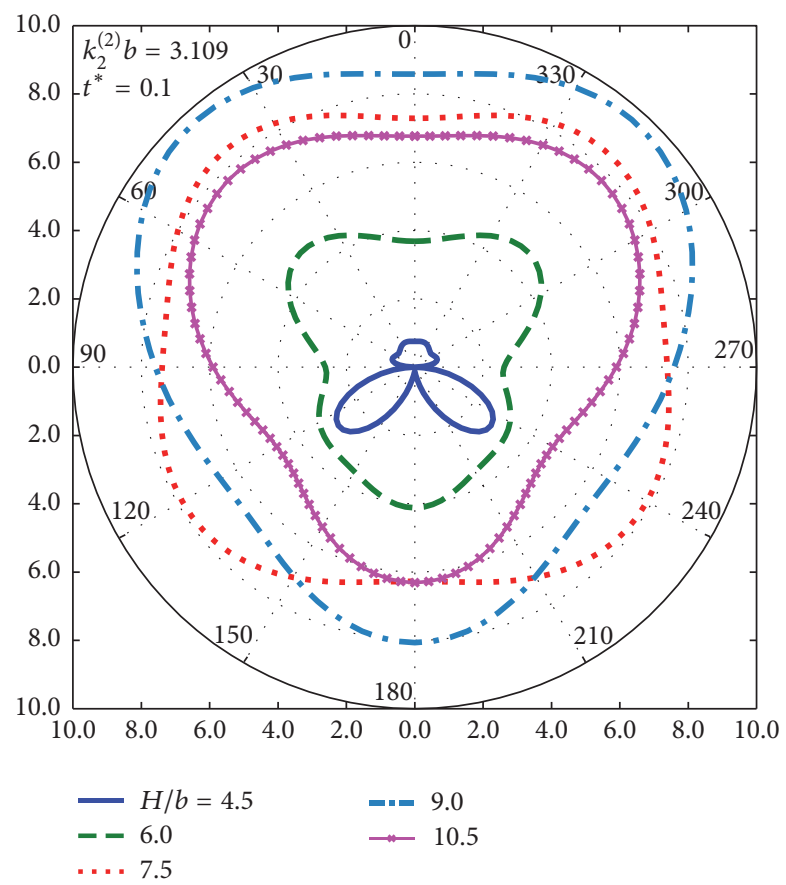

Figure 15: Tangential stress $\left(\sigma_{\theta \theta}^{*}\right)$ versus polar angle at $r=b$ for different depths $(H / b)$ for a tunnel in soft soil.

Note that the distribution becomes nearly symmetrical about the $y$-axis with increasing thickness. As in the case of hard soil, the maximum tangential stress (Figure 17) in soft soil decreases as the thickness increases. However, the point at which the maximum stress occurs varies significantly depending on the values of the thickness of the tunnel. Based

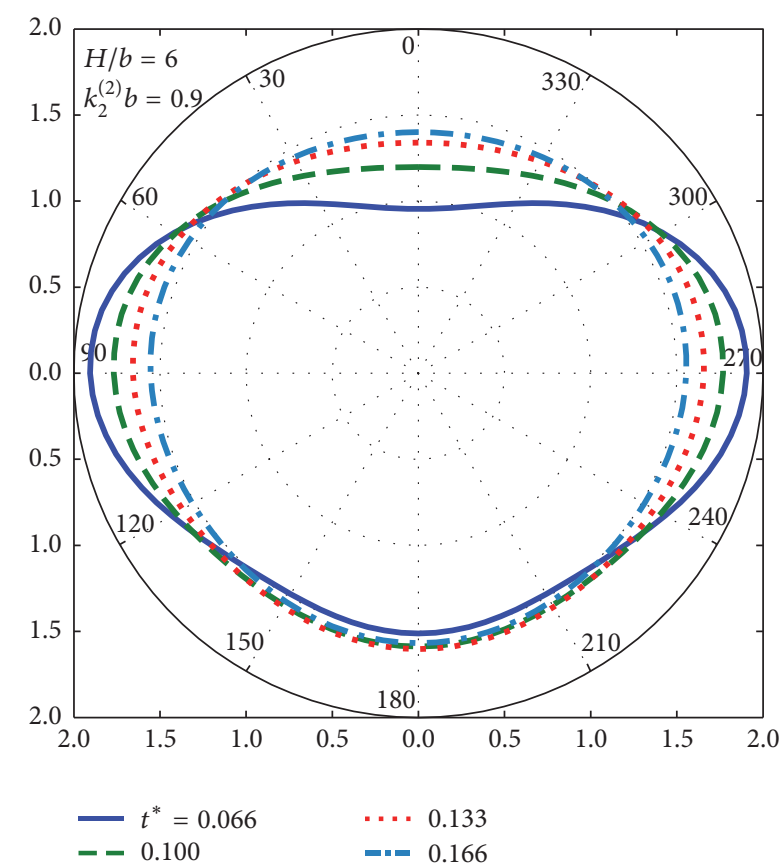

FIgURE 16: Tangential stress $\left(\sigma_{\theta \theta}^{*}\right)$ versus polar angle at $r=b$ for different thicknesses $\left(t^{*}\right)$ for a tunnel in hard soil.

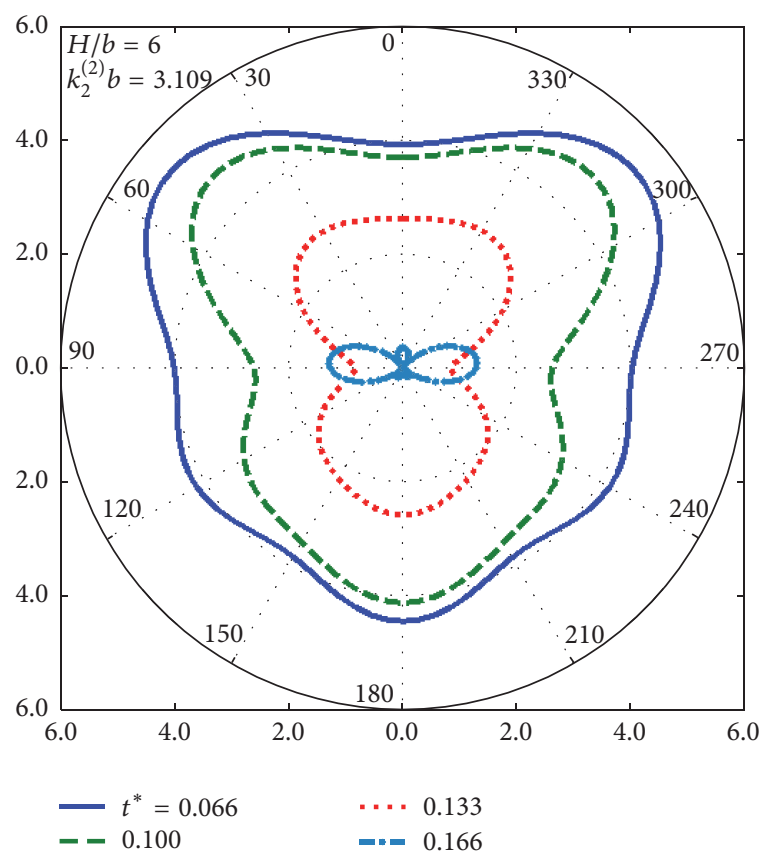

FIgURE 17: Tangential stress $\left(\sigma_{\theta \theta}^{*}\right)$ versus polar angle at $r=b$ for different thicknesses $\left(t^{*}\right)$ for a tunnel in soft soil.

on numerical results (not shown here), the maximum values of the radial stresses and radial and tangential displacements also decreased as the wall thickness increased for both soils. In other words, a thinner tunnel experiences higher stresses and displacements. 


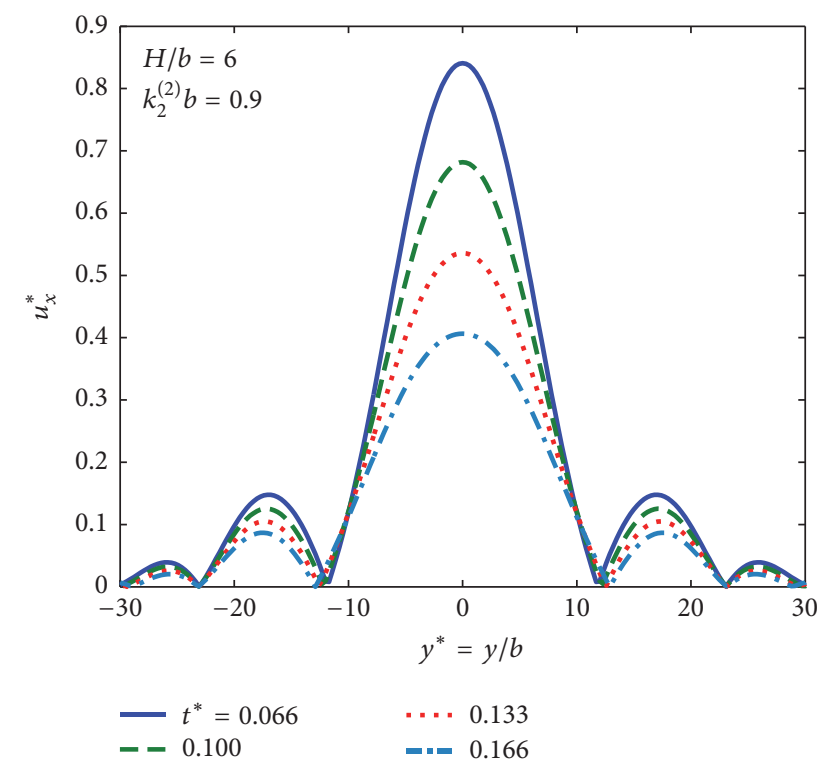

FIgURE 18: Vertical displacement $\left(u_{x}^{*}\right)$ at the free surface of the half space for different thicknesses $\left(t^{*}\right)$ for a tunnel in hard soil.

Finally, the effect of the wall thickness on the free-surface vertical displacements $\left(u_{x}^{*}\right)$ is shown in Figure 18 for the hardsoil case. In this figure, the displacements along the surface of the half space are plotted from $y / b=-30$ to $y / b=30$, where $y / b$ is the dimensionless distance along the horizontal $y$-axis. As expected, the vertical displacements along the free surface decrease as the horizontal distance to the tunnel increases, and the maximum values occur at the point nearest to the top of the tunnel (i.e., $\theta=0^{\circ}$ and $x=H$ ). Furthermore, one can see that free surface displacements decrease as the thickness of the tunnel increases. The corresponding results for the soft soil (not shown here) are similar, but the displacement magnitudes were considerably larger.

\section{Conclusions}

In this paper, the dynamic response of a circular tunnel buried in an elastic half space is discussed. Two types of ground material (hard and soft soil) were considered for the half space for comparison purposes. The effects of the soil type, frequency, and depth on the stresses and displacements at the outer surface of the tunnel wall and the effects of the tunnel thickness on the stresses at the tunnel wall and the vertical displacements at the free surface of the half space have been presented in the figures. It was found that, generally, larger stresses and displacements occurred in the soft-soil case than in the hard-soil case. Additionally, the radial displacements and tangential stresses in both soils were significantly larger than the corresponding tangential displacements and radial stresses, respectively.

In the range of frequencies considered, the maximum radial stresses in the hard soil and the maximum tangential stresses in the soft soil first decreased with frequency and then increased. However, the maximum values of the tangential stress in the hard soil and radial stress in the soft soil increased first with frequency and then decreased. For the hard soil, it was observed that the maximum radial stresses occurred at the points nearest and farthest from the free surface; the maximum tangential stresses occurred at points along the $y$ axis. For soft soil, however, the maximum radial and tangential stresses generally occurred in the upper half of the tunnel. Similar to the variations in maximum stresses, the maximum displacements, in absolute terms, also increased and/or decreased significantly with frequency for both the soils. In particular, the maximum radial displacements occurred at the points nearest and farthest from the free surface.

Variations of stress and displacement at the outer surface of the tunnel wall strongly depended on the depth of embedment. The maximum radial stresses decreased with increasing depth for both soils. The maximum tangential stress increased with depth in the hard soil but increased, decreased, and then increased again in the soft soil. As in the case of the variations with frequency, the maximum displacements (like the maximum stresses) also increased and/or decreased significantly with depth for both soils.

The thickness of the tunnel also affected the stresses and displacements considerably. Both the maximum stress and displacement in the tunnel and the maximum vertical displacement at the free surface (which occurs at the point nearest to the top of the tunnel) decreased as the thickness of the tunnel increased. Additionally, the amplitudes of the vertical displacement along the free surface decreased gradually as one moved away from the tunnel, as expected.

From the preceding remarks, it can be concluded that the properties of the surrounding ground, the frequency, the thickness of the tunnel wall, and the depth of embedment have a considerable effect on the dynamic response of the tunnel.

\section{Conflicts of Interest}

The authors declare that there are no conflicts of interest regarding the publication of this paper.

\section{References}

[1] V. R. Thiruvenkatachar and K. Viswanathan, "Dynamic response of an elastic half space with cylindrical cavity to timedependent surface tractions over the boundary of the cavity," Journal of Mathematics and Mechanics, vol. 14, pp. 541-571, 1965.

[2] N. El-Akily and S. K. Datta, "Response of a circular cylindrical shell to disturbances in a half-space," Earthquake Engineering \& Structural Dynamics, vol. 8, no. 5, pp. 469-477, 1980.

[3] N. El-Akily and S. K. Datta, "Response of a circular cylindrical shell to disturbances in a half-space-numerical results," Earthquake Engineering \& Structural Dynamics, vol. 9, no. 5, pp. 477487, 1981.

[4] S. K. Datta, A. H. Shah, and K. C. Wong, "Dynamic stresses and displacements in buried pipe," Journal of Engineering Mechanics, vol. 110, no. 10, pp. 1451-1466, 1984.

[5] K. C. Wong, A. H. Shah, and S. K. Datta, "Dynamic stresses and displacements in a buried tunnel," Journal of Engineering Mechanics, vol. 111, no. 2, pp. 218-234, 1985.

[6] T. Balendra, C. G. Koh, and Y. C. Ho, "Dynamic response of buildings due to trains in underground tunnels," Earthquake 
Engineering \& Structural Dynamics, vol. 20, no. 3, pp. 275-291, 1991.

[7] V. W. Lee and J. Karl, "Diffraction of SV waves by underground, circular, cylindrical cavities," Soil Dynamics and Earthquake Engineering, vol. 11, no. 8, pp. 445-456, 1992.

[8] J. E. Luco and F. C. P. de Barros, "Seismic response of a cylindrical shell embedded in a layered viscoelastic half-space. I: formulation," Earthquake Engineering \& Structural Dynamics, vol. 23, no. 5, pp. 553-567, 1994.

[9] F. C. P. de Barros and J. E. Luco, "Seismic response of a cylindrical shell embedded in a layered viscoelastic half-space. II: validation and numerical results," Earthquake Engineering and Structural Dynamics, vol. 23, no. 5, pp. 569-580, 1994.

[10] F. Guan and I. D. Moore, “Three-dimensional dynamic response of twin cavities due to traveling loads," Journal of Engineering Mechanics, vol. 120, no. 3, pp. 637-651, 1994.

[11] H. Bayıroğlu, Forced vibrations of structures embedded in halfspace [Ph.D. thesis], İstanbul Technical University, İstanbul, Turkey, 1994 (Turkish).

[12] A. A. Stamos and D. E. Beskos, "3-D seismic response analysis of long lined tunnels in half-space," Soil Dynamics and Earthquake Engineering, vol. 15, no. 2, pp. 111-118, 1996.

[13] C. A. Davis, V. W. Lee, and J. P. Bardet, "Transverse responce of underground cavities and pipes to incident SV waves," Earthquake Engineering and Structural Dynamics, vol. 30, no. 3, pp. 409-410, 2001.

[14] Y. Yang, H. Hung, and L. Hsu, "Ground vibrations due to underground trains considering soil-tunnel interaction," Interaction and multiscale mechanics, vol. 1, no. 1, pp. 157-175, 2008.

[15] J. Liang, Z. Ba, and V. W. Lee, "Diffraction of plane SV waves by an underground circular cavity in a saturated poroelastic halfspace," ISET Journal of Earthquake Technology, vol. 44, no. 2, pp. 341-344, 2007.

[16] L.-F. Jiang, X.-L. Zhou, and J.-H. Wang, "Scattering of a plane wave by a lined cylindrical cavity in a poroelastic half-plane," Computers and Geotechnics, vol. 36, no. 5, pp. 773-786, 2009.

[17] X.-L. Zhou, J.-H. Wang, B. Xu, and L.-F. Jiang, "Dynamic response of a circular pipeline in a poroelastic medium," Mechanics Research Communications, vol. 36, no. 8, pp. 898-905, 2009.

[18] S. Gupta, Y. Stanus, G. Lombaert, and G. Degrande, "Influence of tunnel and soil parameters on vibrations from underground railways," Journal of Sound and Vibration, vol. 327, no. 1-2, pp. 70-91, 2009.

[19] C. H. Lin, V. W. Lee, M. I. Todorovska, and M. D. Trifunac, "Zero-stress, cylindrical wave functions around a circular underground tunnel in a flat, elastic half-space: incident Pwaves," Soil Dynamics and Earthquake Engineering, vol. 30, no. 10, pp. 879-894, 2010.

[20] I. Coşkun, H. Engin, and A. Özmutlu, "Dynamic stress and displacement in an elastic half-space with a cylindrical cavity," Shock and Vibration, vol. 18, no. 6, pp. 827-838, 2011.

[21] Q. Liu and R. Wang, "Dynamic response of twin closely-spaced circular tunnels to harmonic plane waves in a full space," Tunnelling and Underground Space Technology, vol. 32, pp. 212220, 2012.

[22] Q. Liu, M. Zhao, and L. Wang, "Scattering of plane P, SV or Rayleigh waves by a shallow lined tunnel in an elastic half space," Soil Dynamics and Earthquake Engineering, vol. 49, pp. 52-63, 2013.

[23] W. I. Hamad, H. E. M. Hunt, J. P. Talbot, M. F. M. Hussein, and D. J. Thompson, "The dynamic interaction of twin tunnels embedded in a homogeneous half-space," in Proceedings of the 5th ECCOMAS Thematic Conference on Computational Methods in Structural Dynamics and Earthquake Engineering, Crete Island, Greece, May 2015.

[24] J. Huang, M. Zhao, and X. Du, "Non-linear seismic responses of tunnels within normal fault ground under obliquely incident $\mathrm{P}$ waves," Tunnelling and Underground Space Technology, vol. 61, pp. 26-39, 2017.

[25] A. C. Eringen and E. S. Şuhubi, Elastodynamics, vol. 2, Academic Press, New York, NY, USA, 1975. 


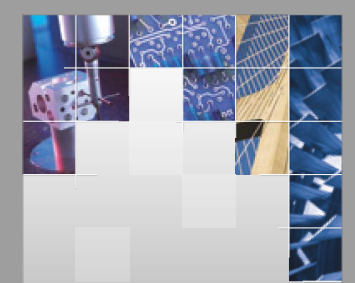

\section{Enfincering}
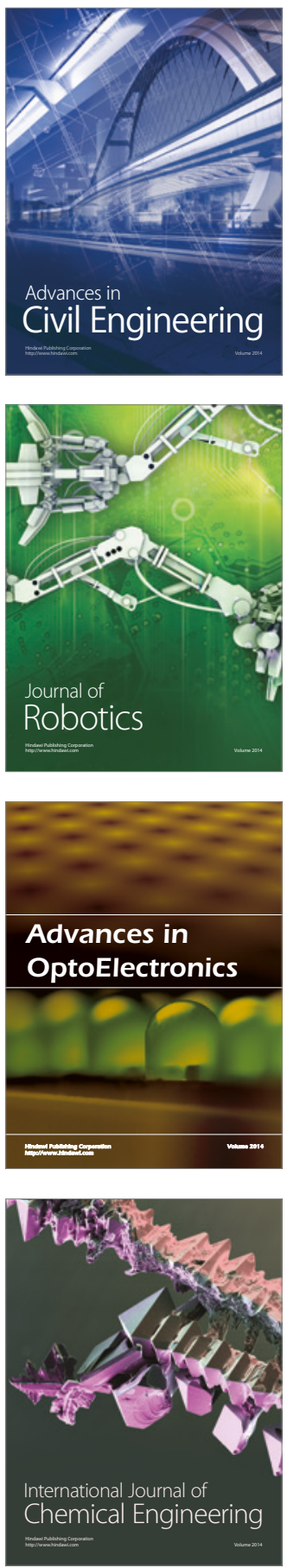

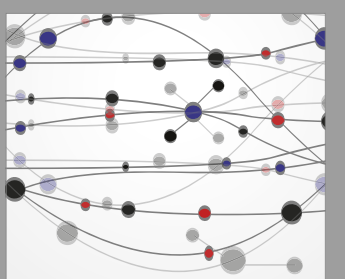

The Scientific World Journal

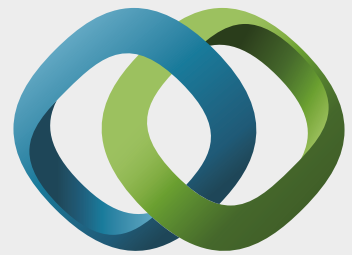

\section{Hindawi}

Submit your manuscripts at

https://www.hindawi.com
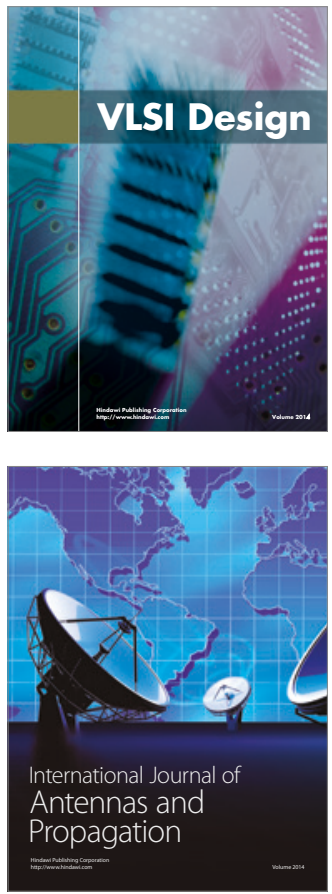

\section{Rotating}

Machinery
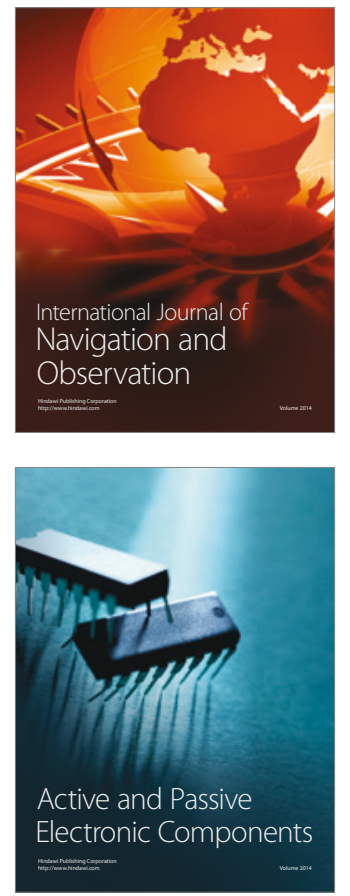
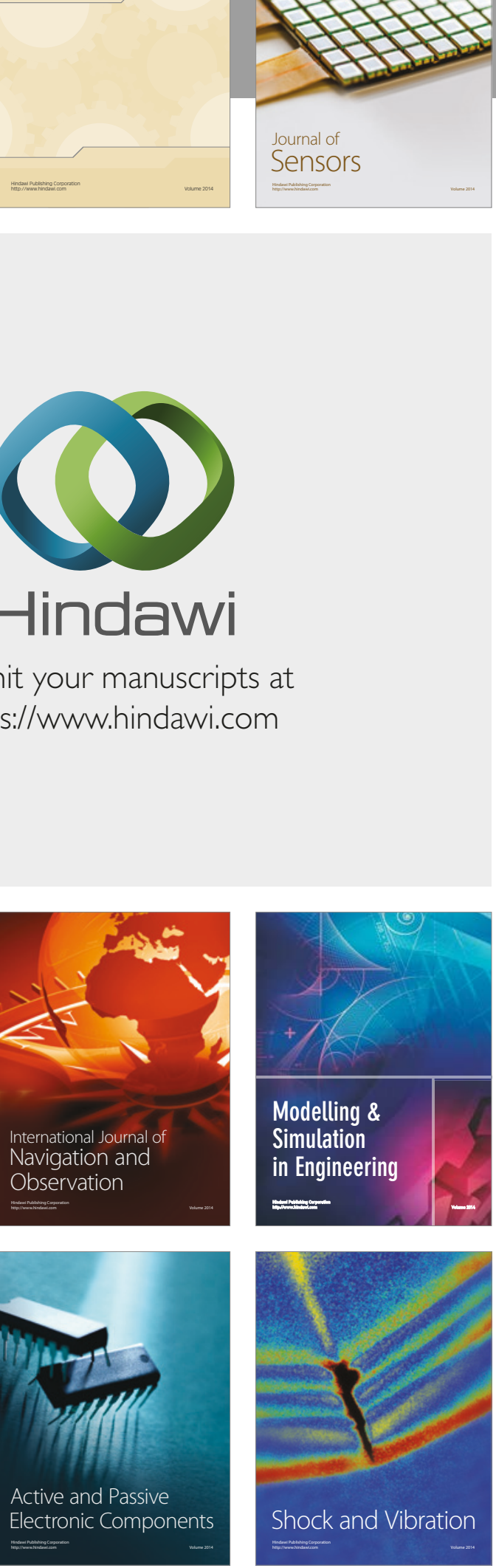
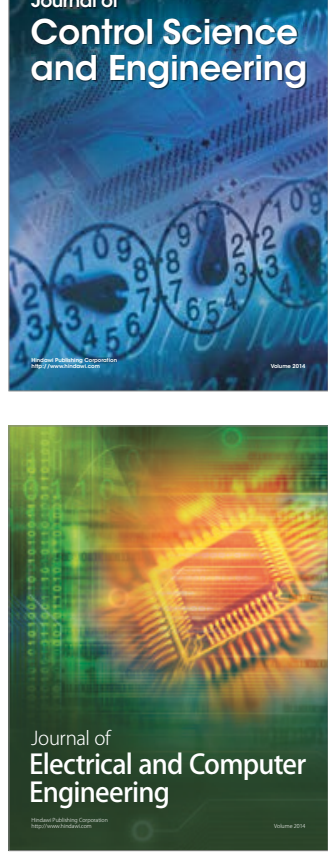

Distributed

Journal of

Control Science

and Engineering
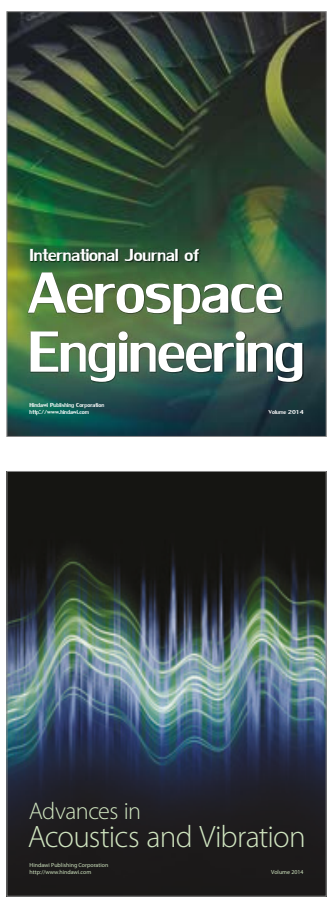

Sensor Networks 